\title{
Long-Term Behaviour of the Variable Wind of P Cygni
}

\author{
Indrek Kolka
}

Tartu Observatory, 61602 Tõravere, Estonia

Introduction. P Cygni has been for a long time an often-used target of observations, and a test object to prove several modelling approaches (cf. e.g. Najarro et al. 1997, and references therein). However, the origin of it's stable variability pattern with moderate amplitudes is poorly known yet. In this contribution I will concentrate on the spectroscopic variability. A more elaborated version of this paper considering many kinds of variability data on $\mathrm{P}$ Cygni will be published elsewhere (Kolka 1998).

The Main Spectroscopic Variability Cycle. The striking phenomenon in the spectra of $\mathrm{P}$ Cygni is the cyclic drift of subsequent individual components through the absorption part of the P Cyg-type profile. This is demonstrated in Fig.1a in the case of three Balmer lines in 1982 (the data adopted from Markova, Kolka 1989). Every new drift-cycle starts when the component with smaller Doppler-shift becomes deeper than it's neighbour.

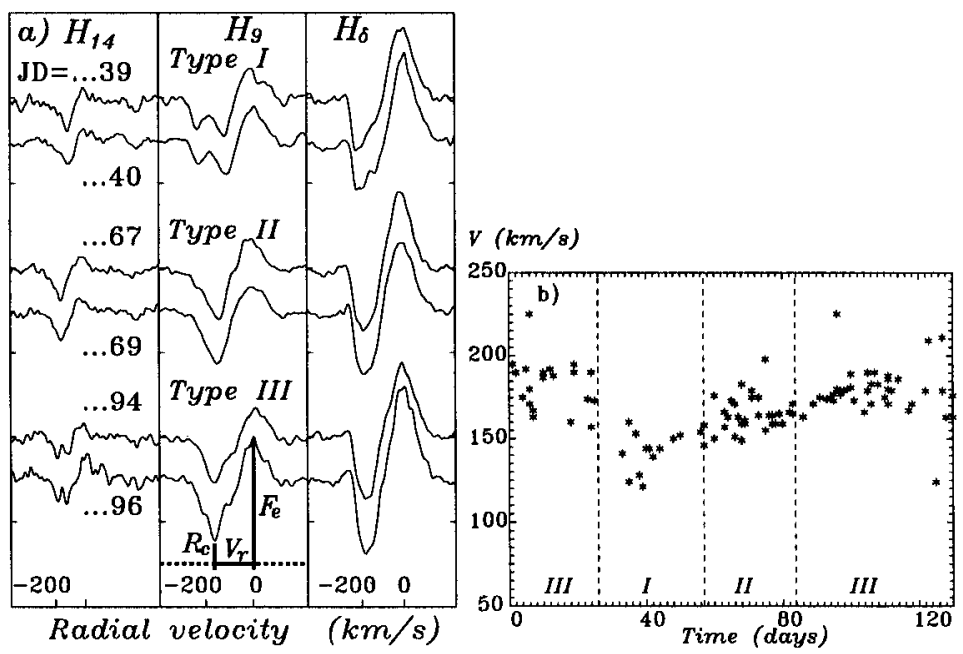

Fig. 1. The drift of components through selected profiles in 1982 (a), and the composite velocity curve of the absorption core in $H_{9}$ (b) 


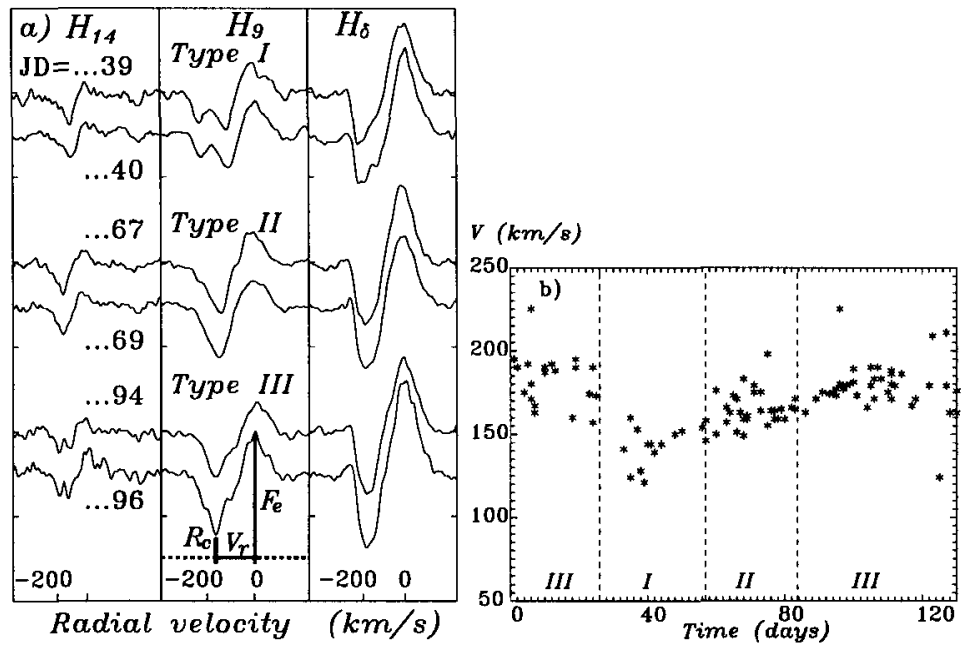

Fig. 1. The drift of components through selected profiles in 1982 (a), and the composite velocity curve of the absorption core in $H_{9}$ (b)

The discussed behaviour is indicative to higher Balmer lines which are sensitive to the line-splitting. Other lines in the optical spectral region are reported to have variable Doppler velocities, too (Markova, Kolka 1989 and Stahl et al. 1995). These lines usually do not exhibit clearly any separable features in the profile but show rather smooth transition between deeper and shallower periods of their shape. To relate the variability in lines of different origin the curves of velocity variations in $\mathrm{H}_{9}$ were completed with similar data on a sample of additional lines which cover different intervals on the velocity scale (Fig.2). The cyclical drift is shown for the period in 1990 when an overlap in photographic (Kolka 1994) and CCD data (Stahl et al. 1995) gave the possibility to use for weak lines more reliable CCD-profiles with high $\mathrm{S} / \mathrm{N}$ - ratio. In Fig.2 one can follow the drift of the velocity perturbation through the profiles from the low to high velocity values. The lower panel in Fig.2 supports the interpretation of the velocity drift: the equivalent width of the purely absorption line (OII 4649) in three specified Doppler-velocity intervals reacts accordingly when the opacity enhancement enters or drifts out of the selected region.

Discussion. We have demonstrated that the main spectroscopic cycle operating in $\mathrm{P}$ Cygni has the characteristic length around $100^{d}$. Similar results were obtained by N. Markova (1998). However, other timescales are not ruled out. The line intensity parametres (absorption depths, emission peaks) show 


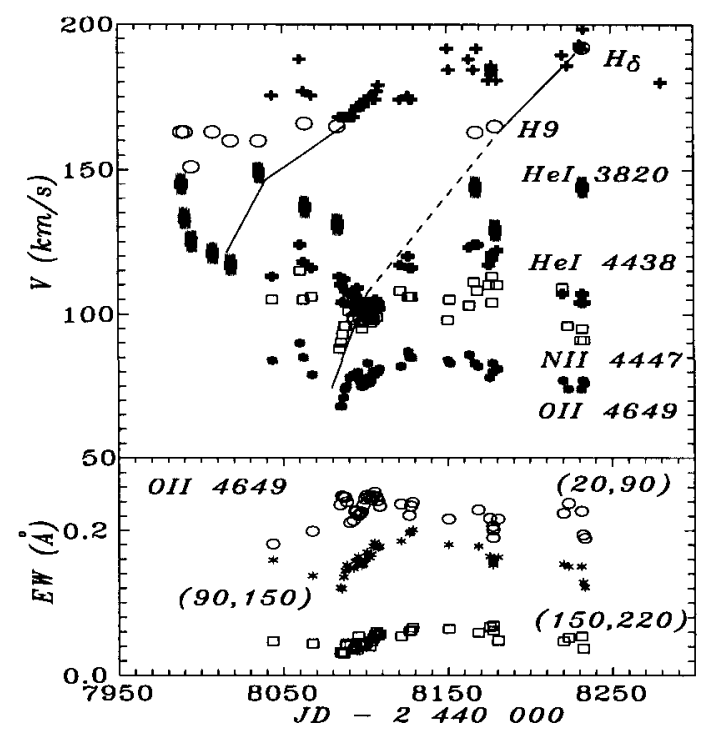

Fig. 2. The drift of the perturbation through different lines, and through different velocity intervals in OII 4649

oscillations both on much longer scales up to $600^{d}$ (Kolka 1994, Markova 1998), and on shorter scales down to $15^{d} \ldots 20^{d}$ (Stahl et al. 1995).

The natural explanation to moving opacity enhancements (to the main spectroscopic cycle) in the line profiles is an expanding enhanced density shell. But the variability on short timescales which is even better exhibited in polarimetric and photometric data (Taylor et al. 1991, Percy et al. 1996) must be interpreted in the limits of the same scenario. The localized inhomogeneities in moving shells are the obvious possibility but their influence on profiles (localized in the velocity!) is not observed.

Another explanation to the $100^{d}$-cycle could be the corotating spiral density-wave - a new popular approach to interpret the cyclical variability. The shape of the wave which is always far from spherical symmetry provides, perhaps, better possibilities to describe rapid variations, too. We notice that the contemporary "best" stellar parametres of P Cygni (see Najarro et al. 1997 ) give to the photospheric rotational period a value excitingly near $100^{d}$ !

\section{References}

Kolka, I. (1994): IAU Symp. 162: Pulsation, Rotation and Mass Loss in Early-Type Stars, eds. L. A. Balona, H. F. Henrichs, J. M. Le Contel, Kluwer, Dordrecht, 536 
Kolka, I. (1998): Baltic Astronomy (submitted)

Markova, N. (1998): poster at this Colloquium

Markova, N., Kolka, I. (1989): Tartu Teated No. 103, 3-15

Najarro, F., Hillier, D. J., Stahl, O. (1997): A\&A,326, 1117

Percy, J. R., Attard, A., Szczesny, M. (1996): A\&AS,117, 255

Stahl, O., Kaufer, A., et al. (1995): The Journ. of Astron. Data, 1, ( CD-ROM)

Taylor, M., Nordsieck, K. H., et al. (1991): AJ, 102, 1197

\section{Discussion}

H. Lamers: In your figure showing the velocity as a function of time, I see as many cases where the velocity decreases with time as those where it increases. Can you explain this?

I. Kolka: This is the natural pattern of the variability cycle. The velocity of the absorption core decreases when the perturbation enters the representative velocity interval, increases thereafter to the highest value and decreases again when the perturbation drifts out of the specified interval.

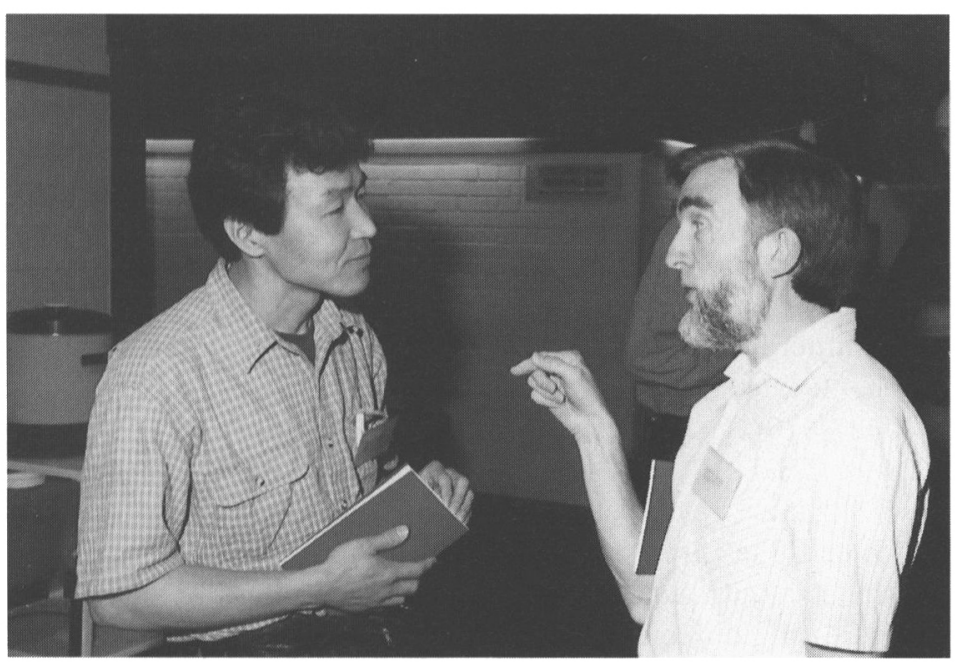

Atsuo Okazaki and Indrek Kolka 